\title{
STRUCTURAL STABILITY OF NEMATIC LIQUID CRYSTAL DROPLETS IN THE LIGHT OF THE THEORY CATASTROPHES
}

\author{
ALEXANDER M. PARSHIN \\ Kirensky Institute of Physics, Federal Research Center KSC SB RAS, \\ Akademgorodok 50, 660036, Krasnoyarsk, Russia \\ Siberian Federal University 79 Svobodny Ave., 660041, Krasnoyarsk, Russia \\ parshin@iph.krasn.ru
}

Received Day Month Day

Revised Day Month Day

\begin{abstract}
Dynamical systems and defects in liquid crystals are described using topological methods. Meanwhile, the director field distribution in liquid crystal droplets is affected by many bulk and surface factors that are difficult to take into account in the topological analysis. Therefore, the structural instability of a liquid crystal droplet formed in a magnetic field has been investigated by us in the framework of the catastrophe theory. The effect of temperature on the control parameters of the cusp catastrophe, which leads to the transition from a bipolar structure with extended poles to the homogeneous or radial configuration, has been examined. It has been established from the potential curves that the transition is induced by the variation in the liquid crystal director orientation between the potential minima related to the liquid crystal polymer anchoring energy and magnetic field. The interplay of the cusp catastrophe control parameters and anchoring parameters has been elucidated.
\end{abstract}

Keywords: Liquid crystal droplet; polymer; anchoring energy; cups catastrophe; control parameters; structural transition.

\section{Introduction}

The theory of catastrophes is a integral part of the theory of dynamical systems. ${ }^{1,2}$ An important characteristic in catastrophe theory is the structural stability of the system. The director field distribution in liquid crystal (LC) structures is affected by both the bulk and surface parameters and their correlation. The mathematical description of these structures refers to the topological methods. In particular, the manifestations of director configurations and their stability around singularities in the nematic layers can be estimated by comparing topological number $n$ corresponding to the number of director revolutions $\boldsymbol{n}$ around disclinations with elasticity constant $K_{\mathrm{ii}}$ characterizing the bulk properties of LCs. ${ }^{3,4}$ However, as was shown in Ref. 5, under special conditions on the LC bounding surfaces the correlation between the bulk and surface parameters can lead to new structural formations, which are difficult to describe by the topological methods. The excellent objects for fine combination of the surface and bulk parameters are LC droplets in polymer matrices. In such droplets, the variation of the boundary conditions can lead to structural transformations. For instance, adding a nematic with a surfactant ${ }^{6}$ allowed establishing the balance between the forces responsible for the normal and tangential LC alignment. Since the temperature dependences of these forces are different, 
the temperature variation led to the transformation of the bipolar droplet configuration to the radial structure. A similar transformation, but with a series of intermediate structures, resulted from a gradual increase in the surfactant concentration in a nematic. ${ }^{7}$ Electric or magnetic field applied to nematic droplets can significantly broaden the range of structural transformations. In particular, the use of electric field together with changing the droplet radius and temperature ${ }^{8}$ facilitated the transformation of the radial structure to the axial one. The electric field applied to radial nematic droplets with a critical radius ${ }^{9}$ led the periodic mutual transformation of the radial and axially symmetric structures. Ref. 10,11 the transitions from the bipolar to radial structure of nematic droplets in magnetic field are observed. The magnetic field was applied to the droplet during its formation and switched off after solidification of the polymer matrix. The transition was accompanied by spontaneous cyclic structural transformations between the bipolar structure with the extended poles and uniform LC alignment in a droplet specified by a magnetic field. However, many bulk and surface factors affecting the transition were impossible to estimate within the topological consideration. In this article the structural stability in nematic droplets formed in a magnetic field in the framework of the catastrophe theory is studied.

\section{Surface energy of a bipolar LC droplet}

The bipolar structure of an LC droplet is illustrated in Fig. 1. The droplet has two poles where the lines of nematic director $\boldsymbol{n}$ converge. The droplet director $\boldsymbol{N}$ passes through the

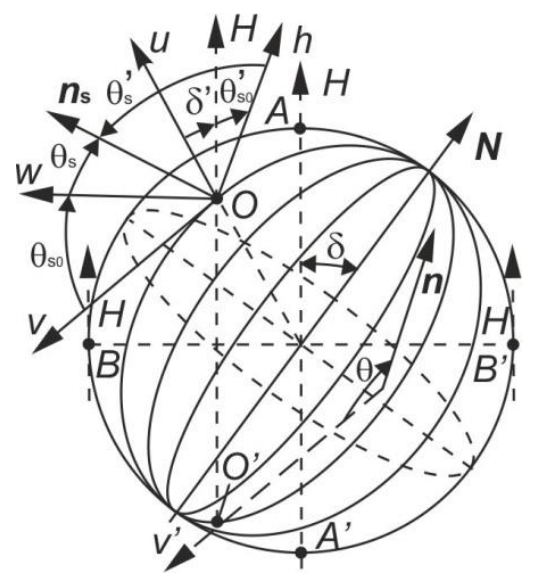

Fig. 1. Schematic of a bipolar droplet: $\boldsymbol{n}$ is the LC director, solid lines show the lines of the surface LC director $\boldsymbol{n}_{\mathrm{s}} ; u$ and $v$ are the axes of local system of coordinates set at arbitrary point $O$ on the droplet surface; $O^{\prime}, A, A^{\prime}$, $B$, and $B$ ' are the characteristic points of application of the $u$ and $v$ axes; $N$ is the droplet director; $w$ and $h$ are the easy orientation axes on the droplet surface that occur under the action of anchoring forces or magnetic field $H ; \theta_{\mathrm{s}}$ and $\theta_{\mathrm{s}}^{\prime}$ are the angles between the $w$ and $h$ axes and director $\boldsymbol{n}_{\mathrm{s}} ; \theta_{\mathrm{s} 0}$ and $\theta_{\mathrm{s} 0}^{\prime}$ are the angles of orientation of the $w$ and $h$ axes relative to the $v$ axis and field $H$; and $\delta$ ' and $\delta$ are the angles of application of magnetic field $H$ relative to the $u$ axis and positioning of $N$ relative to $H$. 
poles and center of the droplet. The $u$ and $v$ axes of the local system of coordinate set at arbitrary point $O$ of the droplet surface are built so that the $u$ axis passed through the droplet center and the $v$ axis was perpendicular to it and lay in the plane containing the point $O$ and droplet poles. The director $\boldsymbol{n}$ at an arbitrary point in the bulk of the droplet makes angle $\theta$ with the $v$ ' axis parallel to the $v$ axis. Anchoring of the LC to the surface specifies the easy orientation axis $w$ directed at angle $\theta_{\mathrm{s} 0}$ to the $v$ axis; the surface director $\boldsymbol{n}_{\mathrm{s}}$ makes angle $\theta_{\mathrm{s}}$ with the $w$ axis. If a droplet is formed in magnetic field $H$ applied at angle $\delta$ ' to the $u$ axis, LC molecules form an imprint on the droplet surface. As a result, after switching off the field, the easy orientation axis $h$ arises, which is directed at angle $\theta$ 's0 to $H$; the surface director $\boldsymbol{n}_{\mathrm{s}}$ deviates by angle $\theta$ 's from this axis. In the general case, the droplet director $N$ makes angle $\delta$ with the $H$ direction.

The free LC energy per unit area of the droplet cavity can be written in the form

$$
\frac{1}{2} F(\theta)=\int_{0}^{R} f_{v}\left(\theta, \theta^{\prime}\right)+F_{s}
$$

where $R$ is the droplet radius, $f_{\mathrm{v}}$ is the volume density of free energy, and $F_{\mathrm{s}}$ is the surface energy. Having generalized the proposed forms of phenomenological representation of $F_{\mathrm{s}}{ }^{12,13}$, we arrive at

$$
F_{s}=-W_{1} \cos \left(\theta_{\mathrm{s}}-\theta_{\mathrm{s} 0}\right)+\frac{1}{2^{n}} \sum_{n=1}^{\infty} W_{2 n} \cos ^{2 n}\left(\theta_{\mathrm{s}}-\theta_{\mathrm{s} 0}\right)+F_{s i},
$$

where $W_{1}$ and $W_{2 n}$ are the energies of LC anchoring to the surface.

The first term $F_{\mathrm{s} 1}=-W_{1} \cos \left(\theta_{\mathrm{s}}-\theta_{\mathrm{s} 0}\right)$ of this expression is caused by the LC polar symmetry breaking near the bounding surface; as a result, molecular dipoles form a polar order parameter that contributes to the surface energy. ${ }^{14}$ The second term is an infinite series sum; however, by now we have experimentally observed only the first contributions to $F_{\mathrm{s}}$ at $n \leq 2$. At $n=1$, this contribution will be $F_{\mathrm{s} 2}=1 / 2 W_{2} \cos ^{2}\left(\theta_{\mathrm{s}}-\right.$ $\left.\theta_{\mathrm{s} 0}\right) .{ }^{15}$ Anchoring energy $W_{2}$ in $F_{\mathrm{s} 2}$ can be presented in the form $W_{2}=\left(W_{0}-W_{\mathrm{E}}\right) .{ }^{16-18}$ The first term in the parenthesis $\left(W_{0}\right)$ is related to the surface tension and steric and dispersion interactions and the second term $\left(W_{\mathrm{E}}\right)$, to the contribution of electric field $E$ induced by the flexoelectric polarization caused by surface charges. At $n=2$, the contribution $F_{\text {s } 4}=1 / 4 W_{4} \cos ^{4}\left(\theta_{\mathrm{s}}-\theta_{\mathrm{s} 0}\right)$ occurs, which was experimentally studied in Ref. 19. As was shown in Ref. 20, this contribution is related to the quadrupole electric polarization caused by the gradient of the order parameter. The last contribution $\left(F_{\mathrm{si}}\right)$ is the isotropic component of the surface energy. ${ }^{21}$

Using Equations 1 and 2, we can obtain the boundary conditions

$$
K \frac{\partial \theta}{\partial u}=\left[W_{1}-\frac{1}{2^{n}} \sum_{n=1}^{\infty} W_{2 n} \cos ^{2 n-1}\left(\theta_{\mathrm{s}}-\theta_{\mathrm{s} 0}\right)\right] \sin \left(\theta_{\mathrm{s}}-\theta_{\mathrm{s} 0}\right),
$$

where $K$ is the modulus of elasticity in the one-constant approximation. ${ }^{22}$

Solving the Equation 3 is a challenging problem, because the LC director distribution $\partial \theta / \partial u$ in the LC droplet at many unknown parameters $\left(W_{1}, W_{2 n}\right)$ is complex. Angles $\theta$ and $\theta_{\mathrm{s}}$ depend on the point of application of the $u$ and $v$ axes on the droplet surface. Therefore, under these conditions, the best variant is to study the extreme points of 
Expression 2 for the surface energy using the catastrophe theory. ${ }^{1,2}$ Dividing Expression 2 by $W_{4}$, we obtain the potential function

$$
V(q)=\frac{1}{4} q^{4}+\frac{1}{2} a q^{2}+b q+c,
$$

where $V(q)=F_{\mathrm{s}} / W_{4}$ is the potential; $a=W_{2} / W_{4}, \quad b=W_{1} / W_{4}$, and $c=F_{\mathrm{si}} / W_{4}$ are the parameters; and $q=\cos \left(\theta_{\mathrm{s}}-\theta_{\mathrm{s} 0}\right)$ is the variable. In the used transformation, as in Expression 2, we limited the number of terms to $n=2$, comparing them with the physical factors at the LC boundary. On the other hand, limitation of Equation 4 to the terms of the order of $O(4)$ in the catastrophe theory is strictly mathematically grounded with the use of the Morse lemma and Thom theorem. The last terms $F_{\text {si }}$ and $V_{0}$ in Equations 2 and 4 can also be ignored for the following reasons. First, the isotropic component $F_{\mathrm{si}}$ is unimportant for the structural transitions, since it is not related to the angles of the deviation of the LC director from the easy orientation axis. Second, nullification of $c$ by ignoring $F_{\text {si }}$ is also correct, since the catastrophe theory deals only with critical points of the $V(q)$ dependence and to take into account the shift of $V(q)$ by a constant $c$ value, it is sufficient to replace the computing origin of the characteristic expression. At $c=0$, Equation 4 turns to the characteristic equation for the cusp catastrophe. Now, the critical values of Equation 4 for any pair of control parameters $a$ and $b$ are determined by differentiation of this equation with respect to $q$

$$
q^{3}+a q+b=0
$$

In contrast to Equation 3, this equation makes it possible to investigate the structural transitions in the LC droplet using standard analysis of the critical points of $V(q)$ function in Equation 4.

To find the roots of cubic Equation 5, it is convenient to use the expression for the discriminant

$$
D=4 a^{3}+27 b^{2}
$$

If the droplet was formed in applied magnetic field $H$, then the potential function is written in the form

$$
V\left(q^{\prime}\right)=\frac{1}{4} d q^{\prime 4}+c^{\prime}
$$

where $d=1 / 4 W_{\mathrm{h}} / W_{4}, W_{\mathrm{h}}$ is the energy of LC anchoring to the molecular imprint formed by magnetic field $H$ on the droplet surface, and $q^{\prime}=\cos \left(\theta^{\prime}{ }_{\mathrm{s}}-\theta^{\prime}{ }_{\mathrm{s} 0}\right)$. In this expression, the fourth power of $q$ ' is determined by the quadratic dependence of the anchoring energy of the molecular imprint $W_{\mathrm{h}}$ on surface order parameter $S_{\mathrm{s}}{ }^{23}$ However, in the case of a droplet, it is necessary to take into account the surface order parameter $S_{\mathrm{ds}}$ caused by the droplet order parameter $S_{\mathrm{d}}$ introduced in Ref. 24. In this consideration, $S_{\mathrm{ds}}$ is a fraction of LC molecules oriented along $h$. At $H=0$, all molecules orient along $w$ and, in the absence of polar forces, arrange tangentially, forming a bipolar structure, in which $S_{\mathrm{ds}} \approx S_{\mathrm{d}}$ $\approx 0.7 .{ }^{24}$ If the $H$ value is sufficiently large to align all molecules along $h$, then we have $S_{\mathrm{ds}}=1$. 


\section{Experiment and results}

The polymer dispersed liquid crystal (PDLC) film was formed using a solution technique described in Refs. 8, 25. Briefly, the 4-n-pentyl-4'-cyanobiphenyl (5CB) nematic with the phase transition sequence $C r-294.6 \mathrm{~K}-N-308.2 \mathrm{~K}-I$ and polyvinylbutyral (PVB) polymer in a weight ratio of 2:3 were dissolved in ethyl alcohol. The prepared solution was poured onto a glass substrate placed between electromagnet poles so that the force lines of magnetic field $H$ acted in the substrate plane. The electromagnet was energized for several hours during which the solvent completely evaporated and a PDLC film formed. After that, the electromagnet was switched off and an ensemble of LC droplets in the film was observed in a polarized-light microscope equipped with a digital camera. Then, the sample was placed in crossed polarizers and illuminated by white light the intensity of which was detected by a CCD matrix. The optical transmittance of each droplet cross section pixel was determined using appropriate software. The PDLC film was formed and investigated at a temperature of $297.2 \mathrm{~K}$; i.e., the LC was in the nematic phase far from the $N-I$ transition temperature.

The PDLC films formed in a magnetic field of $H<4 \mathrm{kOe}$ contained an ensemble of bipolar nematic droplets (Fig. 2). The droplet axes passing through the poles were oriented randomly relative to $H$. As the field was increased $(H>4 \mathrm{kOe})$, the droplets tended to align along the field, so the angle between the droplet axes and $H$ in the ensemble was no larger than $\pi / 4$. In this case, the droplet poles extended or completely vanished and the entire droplet surface became dark. In the field range of
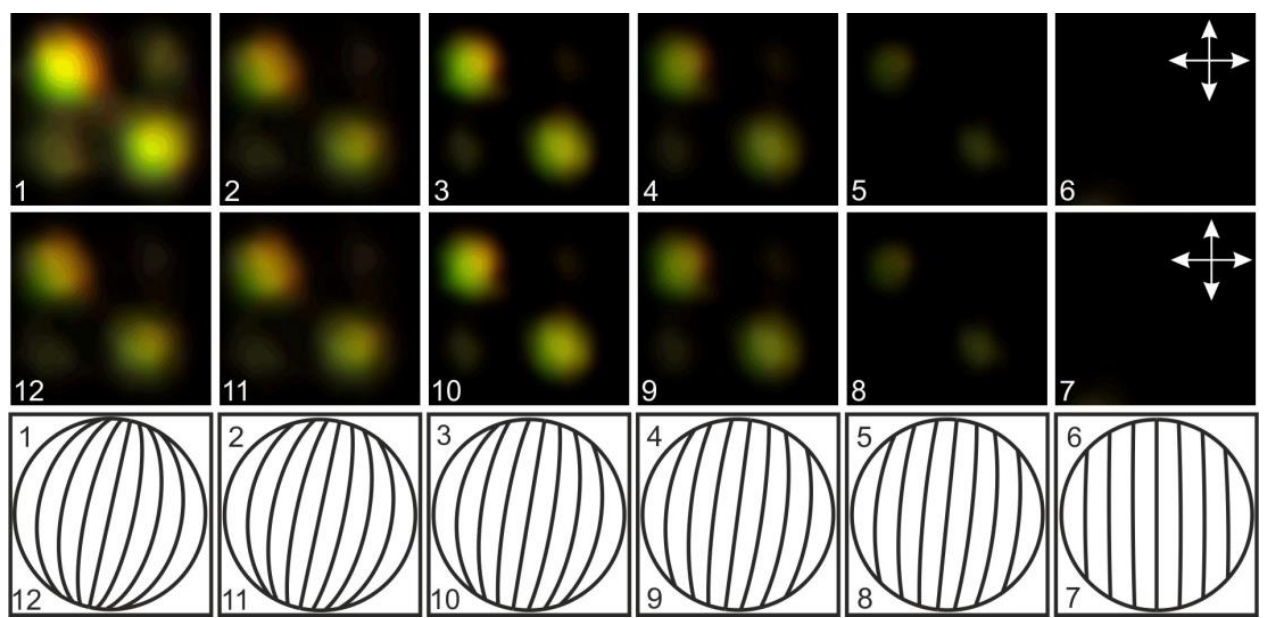

Fig. 2. Microphotograph (at the top) and structure (at the bottom) of a $5 \mathrm{CB}$ droplet $17.5 \mu \mathrm{m}$ in diameter formed in the PVB matrix in a magnetic field of $H=4.5 \mathrm{kOe}$ applied upward. The sequence of frames $1-12$ shows the direction of spontaneous cyclic structural transformations. The interval between frames is $0.15 \mathrm{~s}$. Crossed arrows show the polarizer directions.

$4<H<6$, kOe, spontaneous cyclic transformations occurred, which are illustrated in Fig. 2 (on the top) by a series of frames taken with an interval of $0.15 \mathrm{~s}$ from a droplet 
$17.5 \mu \mathrm{m}$ in diameter at $H=4.5 \mathrm{kOe}$. The transformations were observed as a gradual attenuation in the frame sequence $1 \rightarrow 6$ and backward bleaching in the frame sequence $7 \rightarrow 12$ of the diagonal pairs of four light or dark areas symmetric with respect to the droplet axis. In a magnetic field of $H>6 \mathrm{kOe}$, the poles completely vanished and the bipolar structure transformed to the stable radial structure. ${ }^{8}$

Fig. 3 shows time dependences of optical transmissions $T_{\mathrm{i}}=I_{\mathrm{i}} / I_{0}$, where $I_{\mathrm{i}}$ are the intensities of pixels along the droplet cross section circumferences with diameters $d_{\mathrm{i}}$ $(i=1,2, \ldots 5)$ and $I_{0}$ is the pixel intensity beyond the droplet. The maximum diameter $d_{1}$ is equal to the droplet diameter $d=17.5 \mu \mathrm{m}$. In addition, the average transmission $\Sigma T_{\mathrm{i}}=\Sigma I_{\mathrm{i}} / I_{0}$ is shown.

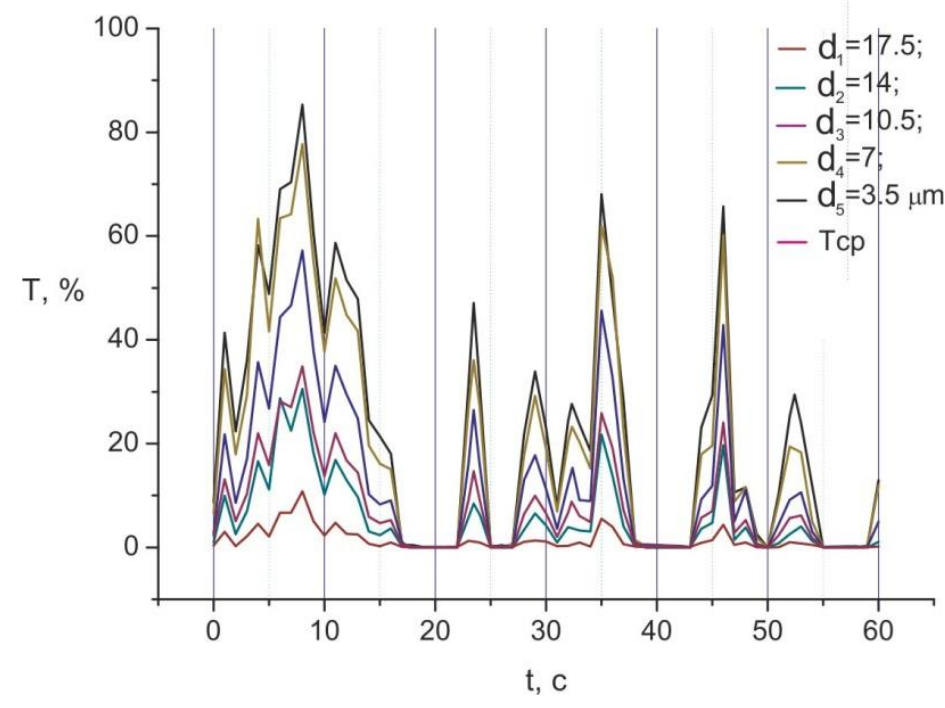

Fig. 3. Time dependence of light transmittance $T$ at droplet diameter cross sections $d_{1}-d_{5} . T_{\mathrm{av}}$ is the average value of $T$.

\section{Discussion}

The existence of the diagonal pairs of bright and dark areas in Fig. 2 is caused by the oblique positioning of the droplet axis with respect to the crossed polarizers. The structural transformations during the cycle can be explained by the uniform extinction or bleaching of the entire droplet, when the dark diagonal areas disappear faster than the bright areas or vice versa. This representation confirms synchronous variation of the optical transmittance $T$ in time for droplets with different cross section diameters (Fig. 3). This optical manifestation is related to rotation of the optical axes of LC molecules in the droplet toward the direction of one of the polarizers, which coincides with the applied magnetic field direction.

Fig. 2 (in the bottom) schematically shows the spontaneous structural transformations of the droplet observed using a polarized-light microscope. ${ }^{25}$ The bipolar structure with extended poles (frames 1/12) transforms to the uniform structure (frames 6/7). 


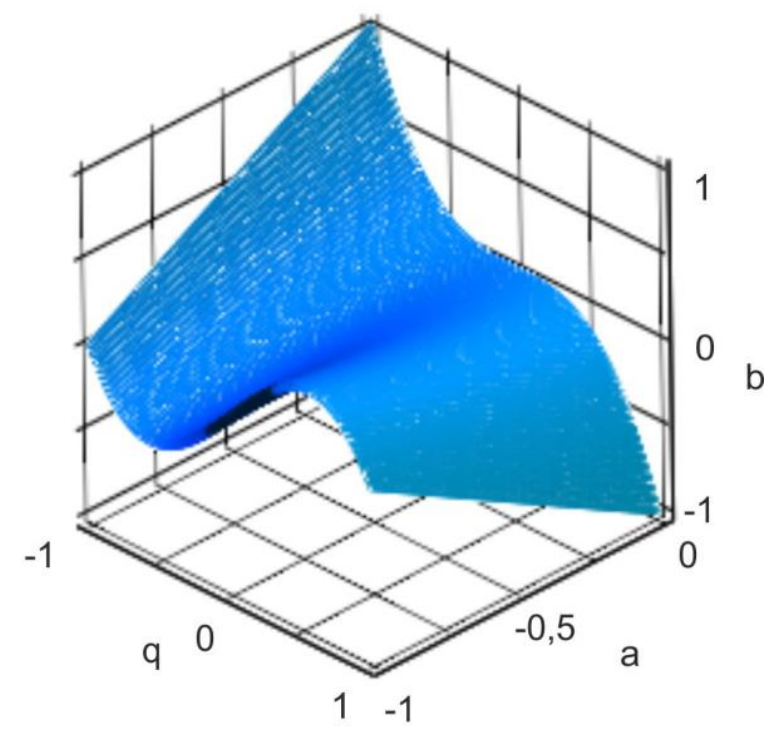

Fig. 4. Cusp catastrophe diversity: $a$ and $b$ are the control parameters and $q$ is the variable.

These transformations are associated with the gradual extension of the droplet poles (frames 2/11-5/8), alignment of the LC director lines, and their rotation toward the direction of the magnetic field $H$ applied during the droplet formation. These transformations can be identified with the rotation of local director $\boldsymbol{n}$ at each point in the bulk of the droplet, rotation of local director $\boldsymbol{n}_{\mathrm{S}}$ on the droplet surface (Fig. 1), and their movement near the poles to direction $h$ specified by the field $H$. In the general case, the structural transformations depend on angle $\delta$ between the droplet director $N$ and $H$ and angle $\delta$ ' between $H$ and the $u$ axis of the local system of coordinates. In this case, the experimentally established fact $\delta<\pi / 4$ related to the trend of the director $N$ to turn along with the $H$ direction during the droplet formation was caused by the nonrigid anchoring of the LC to the gel-like polymer. ${ }^{9}$ In the absence of a magnetic field, the tangential boundary conditions of the 5CB nematic in the PVB matrix and slight ellipticity of a droplet facilitate aligning of the easy orientation axis $w$ along the $v$ axis. Depending on the force of LC anchoring to the droplet surface and point of application of the $u$ and $v$ axes, the angles $\theta^{\prime}{ }_{\mathrm{s} 0}$ between $h$ and $H$ will be different. The $H$ value sufficient to reorient the surface director $\boldsymbol{n}_{\mathrm{s}}$ depends on the angle $\delta^{\prime}$ and is maximum in the diametrically opposite points $A$ and $A^{\prime}$, where $H$ makes an angle of $\pi / 2$ with the $w$ axis; minimum at points $B$ and $B$ ', where the angle between $H$ and $w$ is $\pi$; and has an intermediate value at points $O$ and $O$ '. In view of this, at $\delta<\pi / 4$ the maximum angles $\theta_{\mathrm{s}}$ of the deviation of the surface director $\boldsymbol{n}_{\mathrm{s}}$ for a half-cycle upon structural transformations should be no larger than $\pi / 4$. 
Fig. 4 presents the equilibrium surface built using Equation 5 at arbitrary control parameters $a$ and $b$ and variable $q$. The surface is the manifold of the cusp catastrophe. The catastrophe map is a function determined by expression (6), which represents a set of parameters $a$ and $b$ projected onto the plane (Fig. 5). The curves built at a discriminant of $D=0$ corresponds to bifurcation sets and cross at point 1 , i.e., at the cusp point. Points $1-$ 4 were chosen for characteristic areas of this set.

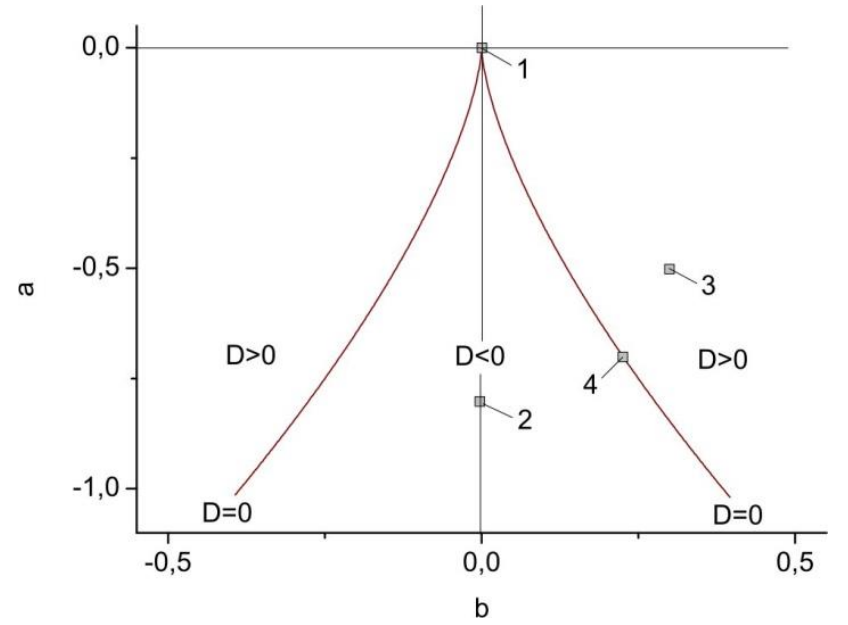

Fig. 5. Set of parameters $a$ and $b$ with characteristic points $1-4$ obtained from (6) in dependence on the values of discriminant $D$. Curves crossing at point 1 are built at $D=0$.

Fig. 6 shows the $V(q)$ curves built using potential function (4) with the $a$ and $b$ values at $c=0$, which correspond to the characteristic points in Fig. 5. Let us consider the case when there are no polar forces and $b=0$. At small negative $a$ values, we obtain curve 1 with the broad minimum. The curve contains cusp point 1 (Fig. 5), which corresponds to three coinciding roots of cubic Equation 5. With increasing negative values $a>-1$, two minima, $-w$ and $w$, with the same potential levels separated by the maximum arise in the $V(q)$ curve symmetrically with respect to the $v$ axis. Curve 1 transforms to curve 2 comparable with the control parameters chosen at point 2 in Fig. 5. This point is located inside the area bounded by the left and right branches of the bifurcation curves and corresponds to three distinct roots of Equation 5. Upon variation of the $a$ value within $-1<a<0$, the positions of the minima change from $q= \pm 0.707$ to $q= \pm 1$, which corresponds to the change in angles $\theta_{\mathrm{s} 0}$ of the deviation of the easy orientation axes $-w$ and $w$ from the $v$ axis from $3 / 4 \pi$ to $\pi$ and from $\pi / 4$ to 0 . At $a<-1$, the $q$ values go beyond the domain of definition. As the $b$ value increases within $0<b<1$ at small negative $a$ values, the positive minimum in curve 1 enhances and rapidly vanishes, while the negative minimum shifts from $q= \pm 0.707$ to $q= \pm 1$ and deepens. This corresponds to the changes in the angle $\theta_{\mathrm{s} 0}$ of the deviation of the easy orientation axis $w$ from the $v$ axis from $3 / 4 \pi$ to $\pi$. Curve 1 passes to curve 3 , which has one minimum and an inflection point (point 3 in Fig. 5). The point was chosen beyond the area bounded by the bifurcation branches and corresponds to one real root in Equation 5. With increasing $a$, the domain of $b$ values at which the $q$ values go beyond the domain of its definition, narrows and completely vanishes at $a>-0.8$ and $b>0.3$. In the narrow 
range of $-0.4<a<-0.9$ at $0.1<b<0.2$, curve 4 is obtained, which contains two minima at different potential levels and has a characteristic point on the right bifurcation branch (Fig. 5). This point corresponds to three roots of Equation 5, the two of which coincide. In the above-mentioned $a$ and $b$ ranges, the minima correspond to the change of angles $\theta_{\mathrm{s} 0}$ of the deviation of the easy orientation axes $-w$ and $w$ from the $v$ axis from $3 / 4 \pi$ to $\pi$ and from $\pi / 4$ to $\pi / 5$. At small positive $a$ values and $b=0$, curve 1 passes to curve 5 with one broad minimum, which is characterized by any point above the straight $a=0$ (Fig. 5). The point corresponds to one real root in Equation 5. As the positive $a$ values increase at $b=0$, curve 5 narrows and tends to form a deep minimum at $a \rightarrow \infty$, which corresponds to one easy orientation axis $w$ oriented normally to the droplet surface along the $u$ axis. With increasing $b$, curve 5 is distorted, its minimum shifts, and the curve becomes similar to curve 3 , but without inflection point. If the magnetic field $H$ is used only at $d>0$ and $c<0$, we can build the $V\left(q^{\prime}\right)$ curve using Equation 7 with the minimum corresponding to curves $1-5$ of the cusp catastrophe. Curve 6 shown in Fig. 6 for $d=0.25$ and $c=-0.15$, which has one broad minimum at $q^{\prime}=0$, satisfies these conditions.

Table 1. Curve numbers corresponding to Fig. 6 in dependence on parameters $a$ and $b$.

\begin{tabular}{|c|c|c|c|c|c|c|c|c|c|c|c|}
\hline $\mathrm{alb}$ & 0 & 0,1 & 0,2 & 0.3 & 0,4 & 0,5 & 0,6 & 0,7 & 0,8 & 0,9 & 1,0 \\
\hline 0 & 1 & 3 & 3 & 3 & 3 & 3 & 3 & 3 & 3 & 3 & 3 \\
\hline$-0,1$ & 1 & 3 & 3 & 3 & 3 & 3 & 3 & 3 & 3 & 3 & $\mathrm{x}$ \\
\hline$-0,2$ & 1 & 3 & 3 & 3 & 3 & 3 & 3 & 3 & 3 & $\mathrm{x}$ & $\mathrm{x}$ \\
\hline$-0,3$ & 2 & 3 & 3 & 3 & 3 & 3 & 3 & 3 & $\mathrm{x}$ & $\mathrm{x}$ & $\mathrm{x}$ \\
\hline$-0,4$ & 2 & 4 & 3 & 3 & 3 & 3 & 3 & $\mathrm{x}$ & $\mathrm{x}$ & $\mathrm{x}$ & $\mathrm{x}$ \\
\hline$-0,5$ & 2 & 4 & 4 & 3 & 3 & 3 & $\mathrm{x}$ & $\mathrm{x}$ & $\mathrm{x}$ & $\mathrm{x}$ & $\mathrm{x}$ \\
\hline$-0,6$ & 2 & 4 & 4 & 3 & 3 & $\mathrm{x}$ & $\mathrm{x}$ & $\mathrm{x}$ & $\mathrm{x}$ & $\mathrm{x}$ & $\mathrm{x}$ \\
\hline$-0,7$ & 2 & 4 & 4 & 3 & $\mathrm{x}$ & $\mathrm{x}$ & $\mathrm{x}$ & $\mathrm{x}$ & $\mathrm{x}$ & $\mathrm{x}$ & $\mathrm{x}$ \\
\hline$-0,8$ & 2 & 4 & 4 & $\mathrm{x}$ & $\mathrm{x}$ & $\mathrm{x}$ & $\mathrm{x}$ & $\mathrm{x}$ & $\mathrm{x}$ & $\mathrm{x}$ & $\mathrm{x}$ \\
\hline$-0,9$ & 2 & 4 & $\mathrm{x}$ & $\mathrm{x}$ & $\mathrm{x}$ & $\mathrm{x}$ & $\mathrm{x}$ & $\mathrm{x}$ & $\mathrm{x}$ & $\mathrm{x}$ & $\mathrm{x}$ \\
\hline-1 & 2 & $\mathrm{x}$ & $\mathrm{x}$ & $\mathrm{x}$ & $\mathrm{x}$ & $\mathrm{x}$ & $\mathrm{x}$ & $\mathrm{x}$ & $\mathrm{x}$ & $\mathrm{x}$ & $\mathrm{x}$ \\
\hline
\end{tabular}

The numbers of curves corresponding to Fig. 6 are given in Table 1 for different parameters $a \leq 0$ and $b \geq 0$. It can be seen that curve 1 is obtained only in a very narrow range of parameters $b=0$ and $-0.2<a<0$. Curve 2 also takes place at $b=0$ and is characterized by a bit broader range of $-1<a<-0.2$. Curve 3 arises upon variation of the parameters between $-0.7<a<0$ and $0<b<1$ and the probability of its occurrence decreases as the $b$ range broadens. Curve 4 also lies in the narrow ranges of $-0.9<a<-0.4$ and $0.1<b<0.2$. The Table 1 is limited to $a=-1$ and $\mathrm{b}=1$, since at $a<-1$ and $\mathrm{b}>1$, the values of variable $q$ go beyond the domain of its definition. Thus, the range of consideration of the control parameters of the cusp catastrophe significantly narrows at their comparison with the parameters describing the surface energy of the interaction of the LC with the surface.

Within the above considerations, the experimental data can be interpreted as follows. In magnetic fields of $H<4 \mathrm{kOe}$, the surface nematic director $\boldsymbol{n}_{\mathrm{s}}$ is oriented along the easy orientation axis $w$, which coincides with the coordinate axis $v$; therefore, the nematic has a bipolar structure. At $H>4 \mathrm{kOe}$, the $w$ axis deviates from the $v$ axis by the angle $\theta_{\mathrm{s} 0}$, which results in the formation of the bipolar structure with the extended poles. The spontaneous structural transformations observed in the range of $4 \mathrm{kOe}<H<6 \mathrm{kOe}$ 
are caused by fluctuations of the nematic director orientation between the minimum of curve 6 (Fig. 6) related to the magnetic field and one of the minima in curves $1-5$ caused by LC anchoring to the droplet surface. It can be seen that the fluctuations are the most probable between the minima in curve 6 and curve 2 corresponding to the $a$ and $b$ values that lie inside the area bounded by the left and right branches of the bifurcation curves

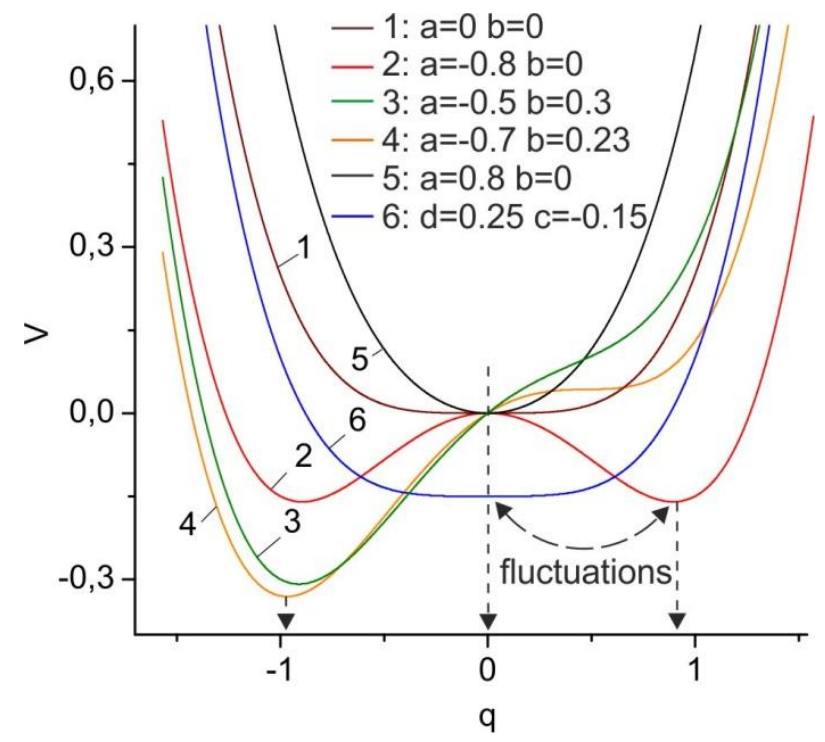

Fig. 6. Characteristic points of the potential functions $V\left(q, q^{\prime}\right)$. Points $1-5$ are obtained from (4) at $a \leq 0$ and $b \geq 0(1-4)$ at points $1-4$ in Figure 5 and at $a>0$ and $b>0(5) ; 6$ is built from (7) at $d>0$ and $c<0$.

(Fig. 5). In addition, the fluctuations can occur between the minima in curve 6 and curve 3 with the $a$ and $b$ values located beyond the bifurcation curve branches; however, the probability of these bifurcations is lower because it is difficult to reach the coincidence of the above-mentioned parameters with parameter $d$. The probability of the fluctuations is even lower between the minimum of curve 6 and the upper or lower minimum of curve 4 , for which $a$ and $b$ lie on the bifurcation curve branches, since in this case the experimental conditions must be matched with the very high accuracy. Let at the initial instant of time the surface director $\boldsymbol{n}_{\mathrm{s}}$ be in the minimum of curve 2, where the variable is $q<1$ and, therefore, the droplet has the bipolar structure with the extended poles (frame 1 in Fig. 2). If the thermal fluctuation energy $k \Omega$, where $k$ is the Boltzmann constant and $\Omega$ is the temperature, appears sufficient to overcome the energy barrier arising at the cross point of potential curves 2 and 6 in Fig. 6 , then the director $\boldsymbol{n}_{\mathrm{s}}$ can pass from the minimum of curve 2 to the minimum of curve 6 (frames $1-6$ in Fig. 2). Then, the director $\boldsymbol{n}_{\mathrm{s}}$ returns from the minimum of curve 6 back to the minimum of curve 2 (frames 7-12 in Fig. 2), which establishes a cycle of structural transformations. It should be noted that the transition between the minima in curves 2 and 6 in Fig. 6 is fast, since it occurs from the narrow energy level. At the same time, the reverse transition occurs with a certain delay. Indeed, as can be seen in Fig. 2, after 1/2 cycle the droplet remains dark for some time (frames 6 and 7) and the optical transmittance in Fig. 3 also has minima $(T=0)$, which remain for some time. This effect can be explained by the fact that the minimum in curve 6 is broad, so a large-scale structural transformation requires accumulation of a certain 
volume of identically oriented molecules on this minimum. In a magnetic field of $H>6 \mathrm{kOe}$, the significant change in the surface order $S_{\mathrm{ds}}$ leads to the flexoelectric polarization, at which the anchoring energy is $W_{2}<0$ and the control parameter is $a>0$. In this case, the transition of the surface director $\boldsymbol{n}_{\mathrm{s}}$ from the minimum of curve 6 to the minimum of curve 5 occurs. Since at the positioning of the coordinate axes $u$ and $v$ at point $A$ in Fig. 1 the $H$ and $u$ directions coincide, the magnetic field-induced uniform orientation of the droplet transforms to the radial structure. Curve 1 can be considered as a particular case of curve 5 .

Based on the above analysis, we can estimate the anchoring energies. As we showed above, the large-scale fluctuations in nematic droplets can be considered using potential curve 2 shown in Fig. 6 . At the $a$ value between $-1<a<-0.2$ given in Table 1 for this curve, the anchoring energy $W_{2}$ at the fluctuations will differ insignificantly from the anchoring energy $W_{4}$ related to the quadrupole electric polarization: $W_{2}=(0.3-1) W 4$. In this case, the anchoring energy related to the surface dipole polarization is $W_{1}=0$, since the control parameter is $b=0$. The effect of surface polarization can also play a certain role, although with the lower probability, in the investigated structural transformations, if the latter will represent fluctuations between the minima in curve 6 and curve 4 with the $a$ and $b$ values of $-0.9<a<-0.4$ and $0.1<b<0.2$ (see Table 1). Then, the anchoring energies $W_{1}$ and $W_{2}$ will be $W_{1}=(0.1-0.2) W_{4}$ and $W_{2}=(0.4-0.9) W 4$, respectively. At $a>0$, the anchoring energy $W_{2}$ will change its sign due to the flexoelectric polarization and can be in any ratio with the anchoring energy $W_{4}$ during the transition of the nematic from the bipolar structure with the extended poles to the radial structure. The same arbitrary ratio with $W_{4}$ will be observed also for the energy $W_{\mathrm{h}}$ of anchoring of the LC molecule imprint on the droplet surface, since upon variation in parameter $d$ only the slope of branches and width of the minimum in curve 6 (Fig. 6) will change at the invariable position of the latter.

\section{Conclusion}

In this article the parameters of LC anchoring to the surface in a bipolar droplet are investigated. These parameters are the anchoring energies $W_{1}, W_{2}$, and $W_{4}$, which are the components of contributions $F_{\mathrm{s} 1}, F_{\mathrm{s} 1}$, and $F_{\mathrm{s} 4}$ to the surface energy $F_{\mathrm{s}}$. The anchoring energy $W_{1}$ is caused by ordering of molecular dipoles at the surface. Parameter $W_{2}$ is characterized by the surface tension, steric and dispersion interactions, and flexoelectric polarization caused by surface charges. Parameter $W_{4}$ is related to the quadrupole electric polarization caused by the order parameter gradient. The investigated anchoring energies were compared with parameters $a$ and $b$ of the potential function $V(q)$ with variable $q$ of the catastrophe theory. When magnetic field $H$ was applied during the LC droplet formation, the energy $W_{\mathrm{h}}$ of nematic anchoring to the molecular imprint specified by field $H$ was introduced. Parameter $W_{\mathrm{h}}$ was compared with parameter $d$ of the potential function $V\left(q^{\prime}\right)$ with variable $q$ '. The 5CB PDLC film was formed by a solution method and the optical transmittance of each droplet cross section pixel was determined with a CCD matrix in crossed polarizers using appropriate software. Time dependences of the optical transmittance of intensities of pixels located on the droplet cross section circumferences 
with different diameters were obtained. The PDLC films formed in a magnetic field of $H<4$ kOe contained an ensemble of bipolar nematic droplets. In the range of $4 \mathrm{kOe}<H<6 \mathrm{kOe}$, spontaneous cyclic structural transformations occurred, which were observed as a gradual attenuation and backward bleaching of the diagonal pairs of four bright or dark areas symmetric with respect to the droplet axis. At $H>6 \mathrm{kOe}$, the poles completely vanished and the bipolar structure transformed to the stable radial configuration. The equilibrium surface obtained by differentiation of the potential function with respect to $q$ was presented for different control parameters $a$ and $b$ and variable $q$. The set of parameters $a$ and $b$ projected onto the plane was presented, which reflects the equilibrium surface and contains the points chosen for characteristic areas of this set. Having compared parameters $a$ and $b$ and variable $q$ of the potential function of cusp catastrophe with the energies of LC anchoring to the surface and angles of nematic director orientation in a droplet, we determined the signs and domains of definition of these parameters. The $V(q)$ curves were obtained from the potential function with the $a$ and $b$ values corresponding to the characteristic points of the mapping set. Using these curves, we determined the range of values of control parameters $a$ and $b$ and domain of definition of variable $q$, as well as the mechanism of structural transformations. At $H<4 \mathrm{kOe}$, the surface nematic director $\boldsymbol{n}_{\mathrm{s}}$ is oriented along the easy orientation axis $w$ parallel to the droplet surface; therefore, the nematic has a bipolar structure. At $H>4 \mathrm{kOe}$, the $w$ axis deviates from the surface, which results in the formation of a bipolar structure with the extended poles. The spontaneous structural transformations observed in the range of $4 \mathrm{kOe}<H<6 \mathrm{kOe}$ are caused by the fluctuations of the nematic director orientation between the curve minimum corresponding to the magnetic field and one of the curve minima caused by LC anchoring to the droplet surface. At $H>6 \mathrm{kOe}$, the control parameter $a$ changes its sign and the surface director $\boldsymbol{n}_{\mathrm{s}}$ passes from the minimum of the curve specified by a magnetic field to the minimum of the curve corresponding to the homeotropic orientation of the nematic. Thus, the transition from the bipolar structure with the extended poles to the radial configuration occurs. Based on the above analysis, the anchoring energies were estimated.

\section{Running Heads}

Structural stability of nematic liquid crystal droplets.

\section{Acknowledgments}

The work was partially supported by the integration project No. 356-2018-0058 of the Siberian Branch of the Russian Academy of Sciences.

\section{References}

1 T. Poston, I. Stewart, Catastrophe theory and its applications (Pitman, London, UK, 1978).

2 J. M. T. Thompson, Instabilities and catastrophes in science and engineering (J. Wiley and Sons, New York, USA, 1982).

3 F. C. Frank, Discuss. Faraday Soc. 25 (1958) 19. 
4 M. Kleman and O. D. Lavrentovich, Phil. Mag. 86 (2006) 4117.

5 A. M. Parshin, V. Y. Zyryanov and V. F. Shabanov, Liq. Cryst. 42 (2015) 57.

6 G. E. Volovik and O. D. Lavrentovich, JETP. 36 (1983) 1159.

7 O. O. Prishchepa, A. V. Shabanov and V. Y. Zyryanov, Phys. Rev. E. 72 (2005) 031712-1.

8 J. H. Erdmann, S. Zumer and J. W. Doane, Phys. Rev. Lett. 64 (1990) 1907.

9 A. V. Koval'chuk, M. V Kurik, O. D. Lavrentovich and V. V. Sergan, JETP 67, (1988) 1065.

10 A. M. Parshin, V. G. Nazarov, V. Y. Zyryanov and V. F. Shabanov, Mol. Cryst. Liq. Cryst. 557 (2012) 50 .

11 A. M. Parshin, V. G. Nazarov, V. Y. Zyryanov and V. F. Shabanov, Crystallography Reports 54 (2009) 1191.

12 G. Barbero, N. V. Madhusudana and G. Durand, Z. Naturforsch. 39a (1984) 1066.

13 G. Barbero and G. Durand, Liquid Crystals in Complex Geometries: Surface Anchoring of Nematic Liquid Crystals, (Taylor and Francis, London, UK, 1996), pp. 21-52.

14 J. D. Parsons, Mol. Cryst. Liq. Cryst. 31 (1975) 79.

15 A. Rapini and M. Papoular, J. Phys. Colloq. 40 (1979) C4-54.

16 G. Barbero and A. G. Petrov, J. Phys.: Condens. Matter. 6 (1994) 2291.

17 V. A. Gunyakov, A. M. Parshin and V. F. Shabanov, Liq. Cryst. 33 (2006) 645

18 V. A. Gunyakov, A. M. Parshin and V. F. Shabanov, Eur. Phys. J. E. 20 (2006) 467.

19 K. H Yang and C. Rosenblatt, Appl. Phys. Lett. 43 (1983) 62.

20 G. Barbero and I. Dozov, J. F. Palierene and G. Durand, Phys. Rev. Lett. 56 (1986) 2056.

21 L. M. Blinov and V. G. Chigrinov, Electrooptic effects in liquid crystal materials (SpringerVerlag, New York, USA, 1996).

22 P. G. de Gennes and J. Prost, The Physics of Liquid Crystals (Clarendon Press, Oxford, UK, 1993).

23 C. Rosenblatt, J. Phys. 45 (1984) 1087.

24 J. R. Kelly and P. Palffy-Muhoray, Mol. Cryst. Liq. Cryst. 243 (1994) 11.

25 P. S. Drzaic, J. Appl. Phys. 60 (1986) 2142. 